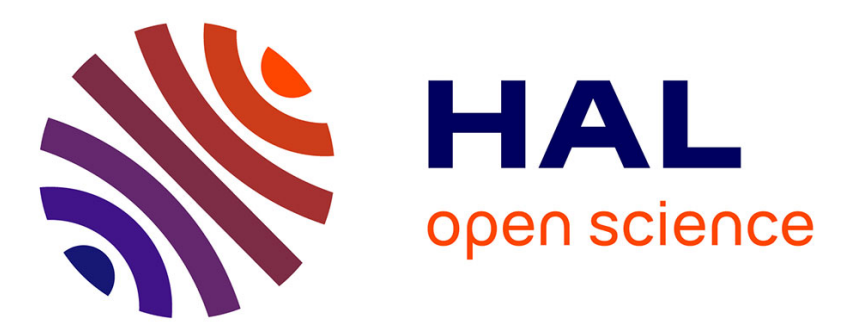

\title{
Localisation de nodules pulmonaires en réalité augmentée grâce au Cone Beam Computed Tomography (CBCT) en vidéo-thoracoscopie
}

Simon Rouzé, Pablo Alvarez, Bertrand de Latour, Erwan Flécher, Jean-Louis Dillenseger, Jean-Philippe Verhoye

\section{- To cite this version:}

Simon Rouzé, Pablo Alvarez, Bertrand de Latour, Erwan Flécher, Jean-Louis Dillenseger, et al.. Localisation de nodules pulmonaires en réalité augmentée grâce au Cone Beam Computed Tomography (CBCT) en vidéo-thoracoscopie. Bulletin de l'Académie Nationale de Médecine, 2018, 202 (8-9), pp.1897-1908. 10.1016/S0001-4079(19)30183-9 . inserm-02319193

\section{HAL Id: inserm-02319193 https://www.hal.inserm.fr/inserm-02319193}

Submitted on 17 Oct 2019

HAL is a multi-disciplinary open access archive for the deposit and dissemination of scientific research documents, whether they are published or not. The documents may come from teaching and research institutions in France or abroad, or from public or private research centers.
L'archive ouverte pluridisciplinaire HAL, est destinée au dépôt et à la diffusion de documents scientifiques de niveau recherche, publiés ou non, émanant des établissements d'enseignement et de recherche français ou étrangers, des laboratoires publics ou privés. 


\title{
Localisation de nodules pulmonaires en réalité augmentée grâce au Cone Beam Computed Tomography (CBCT) en vidéo-thoracoscopie
}

\author{
Mots-clés : Thoracoscopie. Tomographie. Nodule pulmonaire solitaire. Nodules \\ pulmonaires multiples
}

Keywords:Thoracoscopy.Tomography.SolitaryPulmonaryNodule.Multiple Pulmonary Nodules

\author{
Simon ROUZE 1,2,3, Pablo Arthuro ALVAREZ 1, Bertrand de LATOUR 1,2,3, Erwan \\ FLÉCHER ${ }^{1,2,3}$, Jean-Louis DILLENSEGER ${ }^{1}$, Jean-Philippe \\ VERHOYE $1,2,3$
}

\section{Conflits d'intérêt : aucun}

\section{RÉSUMÉ}

Ce travail a pour but d'étudier l'efficacité du CBCT et de son utilisation en réalité augmentée pour localiser des nodules pulmonaires en vidéo-thoracoscopie.

Matériels et Méthodes : Les patients inclus étaient âgés de plus de 18 ans. Ils devaient présenter des lésions pulmonaires uniques ou multiples d'une taille allant de 5 à $20 \mathrm{~mm}$. Ces lésions étaient identifiées en préopératoire grâce à un scanner. La procédure, réalisée sous anesthésie générale, débutait par l'introduction des trocarts de chirurgie et la création d'un pneumothorax. Ensuite était réalisée une acquisition en $C B C T$. La segmentation était ensuite faite permettant d'obtenir une reconstruction $3 D$ de la lésion. Puis, grâce à la réalisation de scopie sur laquelle était projetée la segmentation en réalité augmentée, le nodule était localisé pendant la vidéo-thoracoscopie.

Résultats : De mars 2014 à juin 2018, 25 patients ont été inclus dans notre étude. L'âge moyen était de $63 \pm 11$ ans [37-74 ans]. La taille moyenne des lésions était de $13 \pm 5 \mathrm{~mm}$ [5-20 mm] et leur profondeur moyenne par rapport à la plèvre de $19 \pm 15 \mathrm{~mm}(5-36 \mathrm{~mm})$. La densité moyenne des lésions était de $-5 \pm 170 \mathrm{HU}[-650-250 \mathrm{HU}]$. Quinze patients ont subi un wedge, neuf patients un wedge suivi d'une lobectomie, et un patient une bilobectomie (après conversion en thoracotomie). Le temps opératoire moyen était de $106 \pm$ $42 \mathrm{~min}$ [60-150 min]. Il a été possible de détecter le nodule et de réaliser la résection grâce à la scopie en réalité augmentée chez 24 patients; un nodule n'a pas été détecté du fait d'un mauvais cadrage du CBCT. Le temps moyen de scopie était de $124 \pm 55$ s [24-204 s].

1. Université de Rennes 1, LTSI, Rennes, F-35000, France

2. INSERM, U1099, Rennes, F-35000, France

3 .Service de chirurgie cardio-thoracique et cardio-vasculaire, Centre Hospitalier Universitaire de Rennes, France. 
Le temps moyen entre l'incision cutanée et la localisation du nodule était de $12 \pm 4$ min [7$17 \mathrm{~min}]$.

Conclusion : Ce travail se démarque des techniques actuelles de localisation par son utilisation uniquement per-opératoire en plus de l'utilisation de la réalité augmentée. Le $C B C T$ est un outil efficace pour détecter et aider à la localisation de nodules pulmonaires en chirurgie par vidéo-thoracoscopie. Associé à la réalité augmentée, cet outil offre une avancée significative pour la vidéo-thoracoscopie et la résection de nodules centimétriques non palpables.

\section{SUMMARY}

Objectives: To study the efficiency of CBCT and the use of augmented reality to localize pulmonary nodules in video-assisted thoracic surgery (VATS)

Methods: The patients were 18 yo or older. They were suffering from pulmonary lesions, solitary or multiple, whom size were between 5 and $20 \mathrm{~mm}$. These lesions were identified pre-operatively thanks to CT. These procedures were performed under general anaesthesia. It was followed by trocar insertion and pneumothorax creation. The CBCT acquisition was performed. The segmentation of the nodule, visualized on the CBCT images, was done. It gave us a $3 D$ reconstruction of the nodule. Then, this $3 D$ image was integrated to fluoroscopic images, in augmented reality, performed during the VATS to localize the nodule.

Results: From march 2014 to June 2018, 25 patients have been included in our protocol. Mean age was $63 \pm 11$ yo. Mean size of the lesion was $13 \pm 5 \mathrm{~mm}$ and its depth to the pleura was $19 \pm 15 \mathrm{~mm}$. The mean density of the lesions was $-5 \pm 170 \mathrm{HU}$. Fifteen patients had to undergo a wedge resection, 9 patients a wedge followed by lobectomy and a patient a bi-lobectomy. The mean operative time was $106 \pm 42$ minutes. We have been able to localize the nodule and do its resection in augmented reality for 24 patients. One nodule has not been detected because of a wrong positioning of the field of view of the CBCT. Mean fluoroscopic time was $124 \pm 55$ s. Mean time between skin incision and nodule localization was $12 \pm 4$ minutes.

Conclusion: This work differs from the actual localization technic thanks to its strictly intra-operative approach in augmented reality. The CBCT is an efficient tool to localize pulmonary nodules and is of good held in VATS. Associated with augmented reality, this tool offers a significant progress for nodule localization.

\section{INTRODUCTION}

Avec le développement des programmes de dépistage du cancer bronchopulmonaire par le biais des scanners «low-dose »[1], le nombre de nodules pulmonaires d'histologie indéterminée est amené à croître de manière considérable [2]. Or, la vidéothoracoscopie (VATS) est un outil crucial dans l'obtention de l'histologie de ces lésions $[3,4]$. Les méthodes de guidage des nodules en VATS restent majoritairement préopératoires, invasives, nécessitant une imagerie tomodensitométrique (TDM) additionnelle, avec des taux de complications et de non efficacité du dispositif de guidage non négligeables [5-7]. 
Parallèlement, le Cone Beam Computed Tomography (CBCT) est une modalité d'imagerie qui connaît un véritable essor (implantologie, chirurgie maxillo-faciale, radiothérapie, procédures endo-vasculaires) [8-11]. Cette imagerie rayon X permet d'obtenir des images tridimensionnelles d'une qualité semblable au scanner en résolution spatiale et proche en résolution en contraste. Concernant les pathologies pulmonaires, le CBCT a été étudié en pneumologie interventionnelle, en radiothérapie et en radiologie [12-14]. L'irradiation induite par un CBCT thoracique est en outre moindre qu'un TDM thoracique conventionnel : respectivement 2,3 à $5 \mathrm{mSv}$ versus 4 à $18 \mathrm{mSv}[15,16]$.

Ce travail a pour but d'étudier l'efficacité du CBCT et de son utilisation en réalité augmentée pour localiser des nodules pulmonaires en vidéo-thoracoscopie.

\section{MATÉRIELS ET MÉTHODES}

\section{Critères d'inclusion:}

Les patients inclus dans ce protocole étaient âgés de plus de 18 ans. Ils devaient présenter des lésions pulmonaires uniques ou multiples d'une taille allant de 5 à $20 \mathrm{~mm}$. La nature de ces lésions pouvait être connue (lésion pulmonaire primitive localisation secondaire d'un autre cancer) ou inconnue (chirurgie diagnostique associée ou non à une chirurgie thérapeutique). La distance par rapport à la plèvre de la lésion n'était pas un critère de sélection.

En pré-opératoire, l'identification de ces lésions était réalisée grâce à une TDM thoracique avec des coupes "parenchymateuses » fines $(1,25 \mathrm{~mm})$. Un TEP-scan 18 FDG a également été réalisé, pour mettre en évidence une éventuelle activité hypermétabolique de la lésion à réséquer.

Les patients ont eu un bilan préopératoire conventionnel préalable à une chirurgie thoracique : explorations fonctionnelles respiratoires, avec notamment VEMS - CV +/- DLCO +/- $\mathrm{VO}_{2}$ max ; bilans biologiques (NFS — ionogramme sanguin — bilan d'hémostase - groupe - Rhésus - RAI) ; échographie cardiaque. Les patients ont été informés de la réalisation de la procédure, et ont signé un consentement éclairé.

\section{Critères de non inclusion}

Les patients présentant les caractéristiques suivantes n'ont pas été inclus dans l'étude :

— ré-intervention (antécédent de chirurgie thoracique homolatérale) ;

— incapacité à réaliser ou obtenir une ventilation uni-pulmonaire lors de la chirurgie ;

— personne majeure faisant l'objet d'une protection légale (sauvegarde de justice, curatelle, tutelle) ;

— personne privée de liberté. 


\section{Déroulement de la procédure}

\section{$\underline{\text { Installation }}$}

L'installation du patient lors des acquisitions se faisait en conditions chirurgicales, c'est-à-dire en décubitus latéral (Figure 1). Le positionnement conventionnel (avec appuis) a été modifié au profit de l'utilisation d'un matelas " coquille » à dépression pour autoriser l'acquisition CBCT. En effet, lors d'une acquisition DynaCT, l'ensemble des flancs de la table opératoire devaient être libres, pour autoriser une rotation complète à $211^{\circ}$ de l'amplificateur de brillance $(C$-arm $)$.

En outre, toujours en raison de l'acquisition rotationnelle, les tubulures de l'équipe d'anesthésie ont été sécurisées et les champs opératoires attachés étroitement à la table opératoire.

\section{Acquisition $C B C T$}

Les acquisitions de Cone Beam Computed Tomography étaient obtenues grâce à la fonction DynaCT d'un $C$-arm conçue pour être utilisé en per-opératoire : ils'agissait de l'Artis Zeego System ${ }^{\circledR}$, produit par la société Siemens Healthcare. Celui-ci disposait d'un capteur plan et d'une matrice de 2048×1538 éléments. Les paramètres de notre protocole DynaCT étaient les suivants :

— incrément de l'angle de rotation : $0,5^{\circ}$;

- matrice de $512 \times 512$ voxels ;

$-211^{\circ}$ de trajectoire circulaire — durant 8 secondes ;

— une dose délivrée de 0,36 mGy par coupe en moyenne ;

— un total de 419 projections.

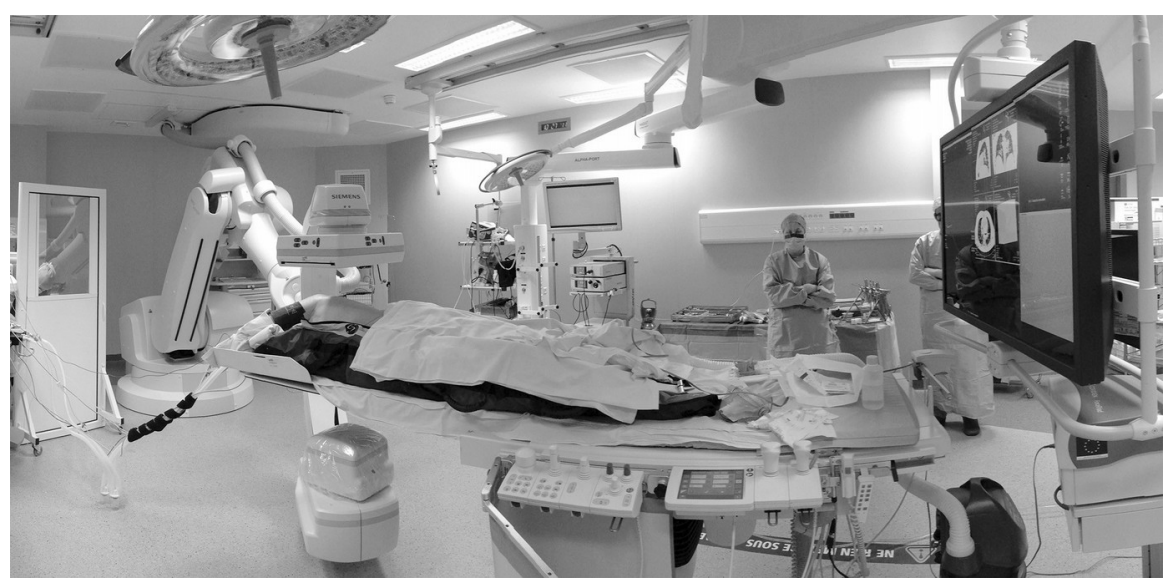

Fig. 1. - Installation du patient : décubitus latéral droit, bras semi pendant, stabilisé par le matelas coquille 
Les images ainsi acquises étaient transférées sur une station de travail dédiée (MMWP station, Siemens Healthcare). Les images DynaCT étaient reconstruites avec une épaisseur de $1 \mathrm{~mm}$, dans les plans axial, coronal et sagittal (Figure 2).

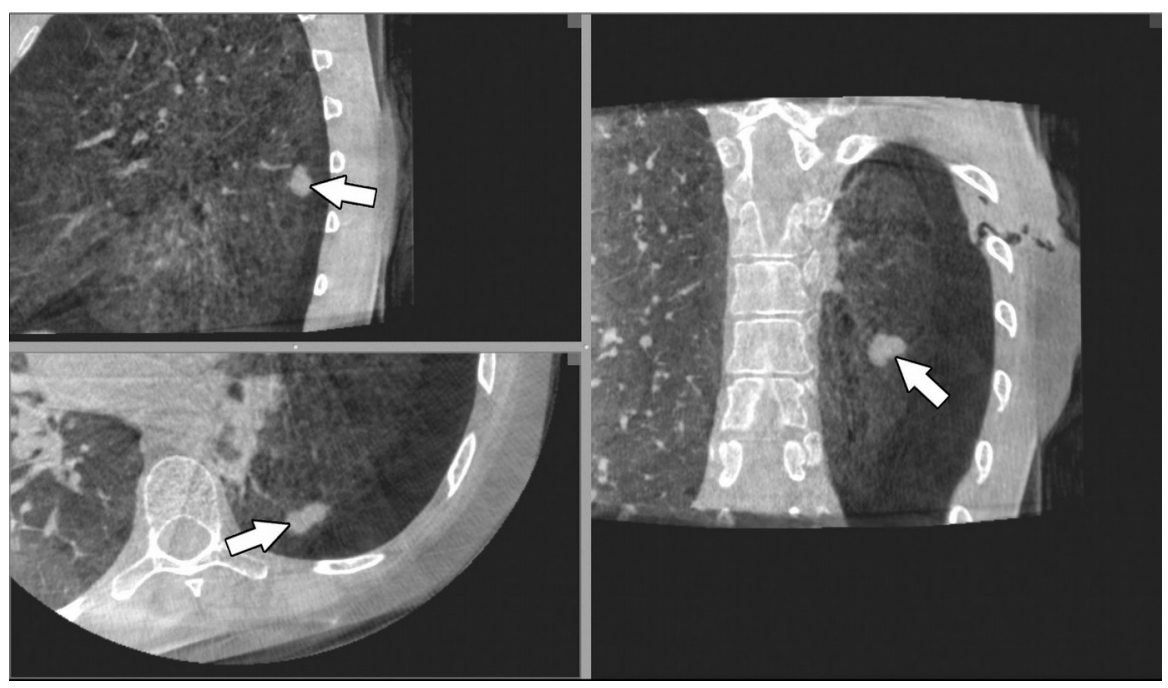

Fig. 2. - Reconstruction multi-planaire à partir du $\mathrm{CBCT}$

Les acquisitions étaient réalisées sur un patient anesthésié, installé, champs opératoires en place, dans des conditions d'asepsie complète. Avant l'acquisition, nous procédions comme suit :

- mise en place du premier trocart de vidéo-thoracoscopie sous contrôle de la vue permettant l'insertion du vidéo-thoracoscope ;

- exclusion du poumon par l'anesthésiste ;

— insufflation d'oxygène (débit entre 1 et $6 \mathrm{~L} / \mathrm{min}$ ) pour permettre un trapping du poumon. En effet, un collapsus trop important du poumon (une atélectasie complète) rendait le parenchyme pulmonaire trop dense pour pouvoir le distinguer de la lésion à repérer;

L'acquisition rotationnelle était ensuite réalisée, poumon controlatéral non ventilé (le temps de l'acquisition seulement).

\section{Procédure chirurgicale}

Le matériel de vidéo-thoracoscopie utilisé était identique au matériel utilisé de manière courante, avec notamment une optique et une colonne Storz HD. La procédure de VATS était réalisée par le biais de trois incisions cutanées permettant unetriangulation. Chacune de ces incisions cutanées autorisait la mise en place d'un trocart par lequel sont introduits les instruments de vidéochirurgie. Une fois la lésion localisée, celle-ci 
était réséquée au moyen d'un système d'agrafage automatique (EndoGIA ${ }^{\circledR}$, Covidien Corporate).

Un examen extemporané était alors réalisé. En fonction de ce dernier, un geste complémentaire était réalisé ou non (curage ganglionnaire médiastinal ou lobectomie + curage ganglionnaire médiastinal).

\section{Segmentation de la lésion et placement du repère}

Une fois les images DynaCT obtenues, le nodule était identifié dans le parenchyme pulmonaire grâce aux reconstructions multiplanaires (MPR). Une fois ce repérage effectué, la lésion était segmentée au moyen d'une segmentation semi-automatique par croissance de région. Enfin, nous utilisions la fonction syngo InSpace EP du logiciel dédié Siemens pour intégrer le volume segmenté au volume de travail.

\section{Localisation peropératoire de la lésion en fluoroscopie et VATS}

Une fois les trocarts en place, l'acquisition DynaCT, le repérage du nodule et la réalisation de sa segmentation réalisés, la procédure de localisation débutait. La lésion segmentée lors de l'étape précédente était localisée dans l'espace, en position absolue, par rapport à la table opératoire. Ainsi, lors d'une acquisition en fluoroscopie conventionnelle, la projection $2 \mathrm{D}$ de cette segmentation était faite de manière automatique, sur l'écran de scopie, associant l'image de la fluoroscopie et l'image de DynaCT segmentée, en temps réel (fonction iPilot Dynamic). En effet, les acquisitions CBCT (et donc la reconstruction du nodule) et le positionnement du $C$-arm (et donc les images de scopie) se faisaient dans le même repère ; la fusion des 2 modalités d'image était donc faite de manière automatique.

Les instruments de vidéochirurgie, sous contrôle de la caméra endoscopique et sous contrôle fluoroscopique, étaient alors introduits. Ils étaient positionnés sur la zone représentant la lésion segmentée, guidés par l'écran de fluoroscopie augmentée. En contrôlant également sur l'écran de vidéo, le parenchyme pulmonaire était alors délicatement saisi, en prenant une attention toute particulière à ne pas mobiliser le poumon. Une seconde acquisition fluoroscopique selon une incidence différente était alors réalisée pour positionner une seconde pince à préhension, permettant une localisation plus précise de la lésion pulmonaire (en gardant toujours un contrôle par l'écran de vidéochirurgie) (Figure 3). 


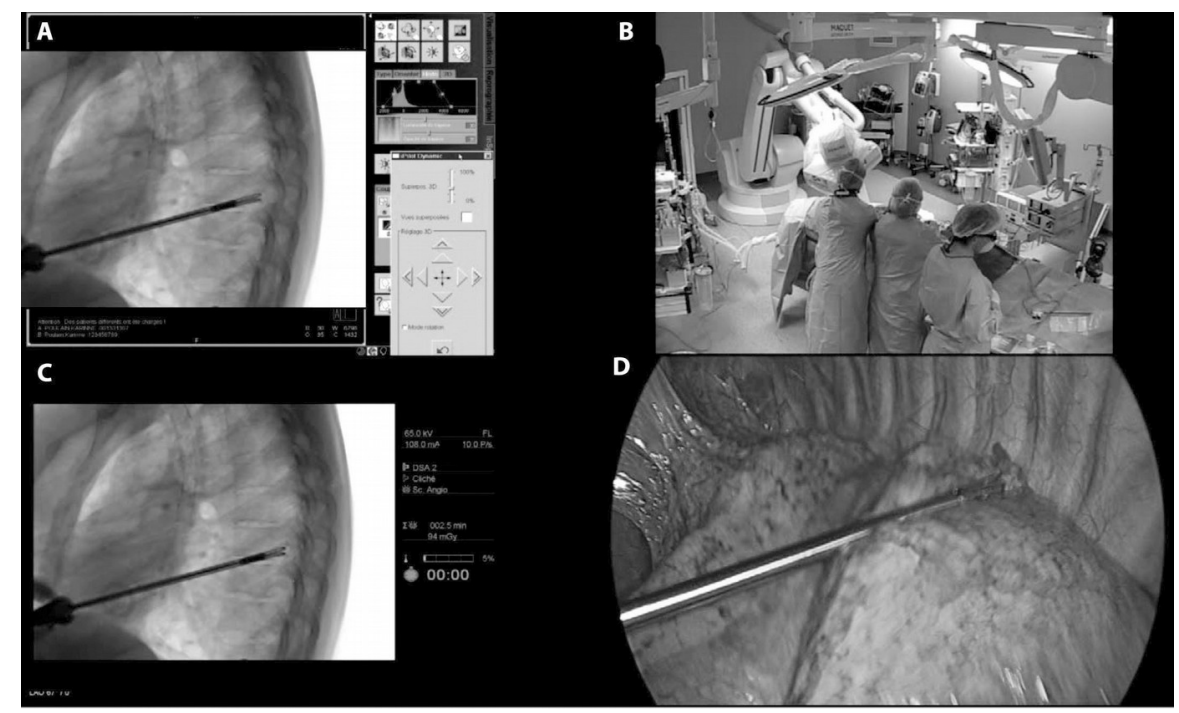

Fig. 3. - procédure de localisation peropératoire avec l'écran de scopie augmentée (en haut à gauche)

\section{RÉSULTATS}

\section{Caractéristiques des patients}

De mars 2014 à juin 2018, 25 patients ont été inclus dans notre étude. L'âge moyen était de $63 \pm 11$ ans [37-74 ans]. Vingt-trois patients avaient une lésion unique et deux patients avaient des lésions multiples. La taille moyenne des lésions était de $13 \pm 5 \mathrm{~mm}$ [5-20 mm] et leur profondeur moyenne par rapport à la plèvre de $19 \pm 15 \mathrm{~mm}(5-$ $36 \mathrm{~mm}$ ). La densité moyenne des lésions était de $-5 \pm 170 \mathrm{HU}$ [-650 -250 HU]. Trois patients présentaient une lésion en verre dépoli. Les caractéristiques des patients sont résumées tableau 1.

Tableau 1. - Caractéristiques des patients

\begin{tabular}{lcl}
\hline & effectif ou moyenne & écart type \\
\hline $\mathrm{N}^{\circ}$ patients & 25 & \\
& & \\
Age $(\mathrm{ans})$ & 63 & \pm 11 \\
$\mathrm{IMC}\left(\mathrm{kg} / \mathrm{m}^{2}\right)$ & 28 & \pm 6 \\
Taille de la lésion $(\mathrm{mm})$ & 13 & \pm 5 \\
Distance à la plèvre $(\mathrm{mm})$ & 19 & \pm 15
\end{tabular}




\begin{tabular}{lll}
\hline Densité (HU) & -5 & \pm 170 \\
\hline
\end{tabular}

\section{Geste chirurgical}

Quinze patients ont subi un wedge. Neuf patients un wedge qui a été suivi d'une lobectomie. Un patient, après la localisation peropératoire (lésion très profonde, proche de l'artère pulmonaire), devait subir une lobectomie. Mais du fait d'une plaie artérielle, il a subi une bi-lobectomie après conversion en thoracotomie. Le temps opératoire moyen était de $106 \pm 42 \mathrm{~min}$ [60 - $150 \mathrm{~min}$ ]. La nature anatomopathologique des lésions était une tumeur primitive pulmonaire (adénocarcinome invasif, adénocarcinome lépidique ou carcinome épidermoïde) pour 11 patients, des métastases pour 8 patients, une tumeur carcinoïde typique pour un patient, une localisation pulmonaire d'une leucémie pro-lymphocytaire et des lésions bénignes pour 3 patients. Un patient a été biopsié d'une lésion bénigne, qui était située à proximité de la lésion maligne qui n'a pas été localisée avec succès. Les 24 autres résections étaient R0.

\section{Acquisition CBCT peropératoire}

L'acquisition $\mathrm{CBCT}$ a été réalisée chez tous les patients. La lésion d'intérêt a pu être identifiée pour 24 malades (96\%). Pour 2 patients l'acquisition DynaCT a néanmoins due être répétée. Les raisons de cette répétition étaient les suivantes :

- pneumothorax trop important, entraînant un collapsus et une densificationt rop importante du parenchyme pulmonaire ; la distinction entre le nodule et le parenchyme sain était alors impossible ;

- reventilation inattendue du poumon exclus et mauvais cadrage du thorax.

Pour un patient, la lésion n'a pas été correctement cadrée dans le champs du CBCT conduisant à une mauvaise localisation.

L'estimation de l'irradiation induite par la procédure était de $3960 \pm 1845 \mu \mathrm{Gy} . \mathrm{m}^{2}$ [821-8283 $\left.\mu \mathrm{Gy} \cdot \mathrm{m}^{2}\right]$.

Plusieurs paramètres ont influé sur la qualité des images :

- la morphologie des patients : les patients minces avaient une meilleure résolution en contraste ;

- le trapping optimal du poumon avec un pneumothorax ni trop important ni trop faible.

La segmentation a été réalisée avec succès pour l'ensemble des patients.

\section{Localisation peropératoire avec scopie en réalité augmentée}

Nous avons été capable de détecter 24 des 25 nodules (96\%) et réaliser la résection grâce à la scopie en réalité augmentée. Le temps moyen de scopie était de $124 \pm 55 \mathrm{~s}$ 
[24-204 s]. Le temps moyen entre le début de l'acquisition des images et la localisation du nodule était de $12 \pm 4 \mathrm{~min}$ [7-17 $\mathrm{min}]$.

\section{Suites opératoires}

Tous les patients sont vivants et sont sortis de l'hôpital. La durée moyenne d'hospitalisation était de $5.0 \pm 4,1$ jours [4-15 j]. Un patient a présenté une pneumopathie post-opératoire avec un bullage prolongé et un autre a présenté une $\mathrm{AC} /$ FA paroxystique après la procédure. Le patient pour lequel la localisation a échoué a dû être réopéré à 1,5 mois (sans conséquence sur le contrôle de sa maladie cancéreuse).

\section{DISCUSSION}

À ce jour et à notre connaissance, ce travail est le premier décrivant l'utilisation peropératoire du CBCT lors d'une chirurgie thoracique. Cette étude montre que le CBCT est un outil utilisable et pertinent pour la localisation peropératoire des nodules pulmonaires avec le concours de la réalité augmentée. En effet, nous avons montré que l'utilisation du CBCT peropératoire était réalisable et permettait d'obtenir des images sur lesquelles les nodules pulmonaires sont identifiables. Ces résultats ont d'ailleurs déjà été démontrés en pneumologie interventionnelle, en radiothérapie, en radiologie, mais jamais lors d'une chirurgie thoracique [12-14,17]. Les interventions chirurgicales étaient d'une durée conventionnelle, en incluant le temps d'acquisition CBCT et de localisation du nodule. Il n'y a eu aucune complication directement liée à la procédure de localisation. Un patient a dû subir une bi-lobectomie à la place d'une lobectomie à cause d'une plaie artérielle. De plus, l'irradiation additionnelle liée à la procédure est acceptable, représentant la moitié d'un scanner conventionnel.

En chirurgie thoracique, Ohtaka et al. ont associé la technologie CBCT à la VATS. Néanmoins, l'utilisation du CBCT est, en réalité, réalisée en pré-opératoire pour placer un hameçon; de plus ils utilisent un O-arm et non un C-arm [17]. En outre, il existe dans cette étude un taux élevé de fistules broncho-pulmonaires (3 fistules sur 10 cas rapportés). Dans le même ordre d'idée, d'autres travaux ont rapporté l'utilisation du $\mathrm{CBCT}$ en chirurgie thoracique, mais il s'agissait à chaque fois d'une utilisation avant l'initiation de la chirurgie pour mettre en place un marqueur [18-20]. En revanche, dans un travail préliminaire (expérimentation animale), Schafer et al. décrivent une approche similaire à la nôtre. Ils combinent l'acquisition $\mathrm{CBCT}$, un recalage automatique, un système de guidage optique et de la réalité augmentée [21]. Ainsi, toutes les informations sont apparemment représentées sur l'écran de vidéothoracoscopie.

Trois patients présentant une lésion en verre dépoli ont été inclus dans l'étude. Leur lésion a été identifiée avec une certaine difficulté sur les images CBCT. En effet, la densité du parenchyme pulmonaire sain est de $\not 900 \mathrm{HU}$, comparé à $€ 300$ jusqu'à $\Varangle 650 \mathrm{HU}$ pour les nodules en verre dépoli. Bien que la différence de contraste en 
résolution du CBCT et du scanner conventionnel ne soit que de 5HU, le pneumothorax et l'affaissement du poumon induisent sa densification. Ainsi, la densité du parenchyme sain et celle du nodule en verre dépoli tendent à se confondre, et c'est aussi grâce aux structures adjacentes (artères, veines et bronches) que la localisation a été possible. C'est pourquoi nous travaillons en parallèle au développement d'un procédé de recalage entre l'imagerie CT préopératoire et le CBCT peropératoire.

En comparaison aux autres systèmes de localisation actuellement disponible, l'utilisation de notre système utilisant le CBCT et la réalité augmentée présente de réels avantages. Il s'agit d'une procédure courte, non invasive, pouvant être réalisée par le chirurgien en personne au bloc opératoire. Le bénéfice en terme organisationnel - quand on sait la difficulté d'organiser les ponctions préopératoires — est non négligeable.

Le patient pour lequel la localisation a échoué met en évidence une des limites de l'utilisation des images CBCT : il s'agissait d'une lésion localisée sur la face médiastinale du poumon à proximité du diaphragme. En plus des difficultés de cadrage des lésions bas situées (ascension du diaphragme chez les patients obèses sous anesthésie générale), nous sommes confrontés à une densification parfois considérable $\mathrm{du}$ parenchyme pulmonaire avec le pneumothorax. Cette densification est particulièrement visible sur la face médiastinale du poumon par simple gravité (zone d'appui en décubitus latéral). Cette donc dans cette zone densifiée que nous avions localisé à tort une lésion bénigne qui était située à proximité de la lésion maligne qui n'était pas dans le champ du CBCT.

Il existe d'autres limites à l'utilisation de ce procédé. Tout d'abord les propriétés biomécaniques exactes du parenchyme pulmonaire ne sont pas encore bien connues : grande déformabilité en périphérie, rigidité près des régions hilaires et de l'arbre trachéo-bronchique. En outre, le poumon est un organe qui est très mobile, plus « plastique » qu' « élastique », avec une vraie mémoire de forme lorsqu'il est non ventilé. De plus, l'acquisition CBCT du poumon est réalisée dans une certaine conformation (position, déflation). Dès lors que le poumon est mobilisé, cette acquisition CBCT n'est plus valide. Aucune solution simple n'existe à l'ensemble de ces difficultés. Une partie des réponses a été proposée par Uneri et al. [22]. Ils décrivent le comportement du poumon après création du pneumothorax à partir de CBCT réalisés en conditions chirurgicales. Néanmoins ce travail apporte une méthode de recalage extrêmement compliquée, dont l'application clinique est clairement limitée par la complexité du modèle mathématique utilisé.

Une autre limite de cette étude réside dans le fait qu'elle a été réalisée dans une salle hybride. Ce type de salle n'est pas utilisé en pratique courante en chirurgie thoracique. En effet, elles sont présentes le plus souvent dans des centres de recherche et leur coût limite de manière importante l'applicabilité de ce travail. De plus, ces salles sont souvent dédiées aux équipes de cardiologie, radiologie, chirurgie cardiaque et vasculaire. Trouver des disponibilités additionnelles aux équipes de chirurgie 
thoracique n'est pas chose facile. Ces limites vont probablement disparaître avec le développement de $\mathrm{C}$-arm équipés de $\mathrm{CBCT}$, plus conventionnels et mobiles [21].

\section{CONCLUSION}

Ce travail innovant et unique se démarque des techniques actuelles de localisation par son utilisation peropératoire associée à l'utilisation de la réalité augmentée. Cette technique est un outil efficace pour détecter et aider à la localisation de nodules pulmonaires en VATS. Associé à la réalité augmentée, cet outil offre une avancée significative pour la vidéo-thoracoscopie et la résection de nodules centimétriques non palpables.

\section{RÉFÉRENCES}

[1] National Lung Screening Trial Research Team, Aberle DR, Adams AM, Berg CD, Black WC, Clapp $\mathrm{JD}$, et al. Reduced lung-cancer mortality with low-dose computed tomographic screening. N Engl J Med 2011;365:395-409. doi:10.1056/NEJMoa1102873.

[2] Pinsky PF, Berg CD. Applying the National Lung Screening Trial eligibility criteria to the US population: what percent of the population and of incident lung cancers would be covered? J Med Screen 2012;19:154-6. doi:10.1258/jms.2012.012010.

[3] Suzuki K, Nagai K, Yoshida J, Ohmatsu H, Takahashi K, Nishimura M, et al. Video-assisted thoracoscopic surgery for small indeterminate pulmonary nodules: indications for preoperative marking. CHEST J 1999;115:563-8.

[4] Luh S, Liu H. Video-assisted thoracic surgery - The past, present status and the future. J Zhejiang Univ Sci B 2006;7:118-28. doi:10.1631/jzus.2006.B0118.

[5] Zaman M, Bilal H, Woo CY, Tang A. In patients undergoing video-assisted thoracoscopic surgery excision, what is the best way to locate a subcentimetre solitary pulmonary nodule in order to achieve successful excision? Interact Cardiovasc Thorac Surg 2012;15:266-72. doi:10.1093/icvts/ivs068.

[6] Tamura M, Oda M, Fujimori H, Shimizu Y, Matsumoto I, Watanabe G. New indication for preoperativemarkingof smallperipheralpulmonarynodulesinthoracoscopicsurgery.Interact Cardiovasc Thorac Surg 2010;11:590-3. doi:10.1510/icvts.2010.241018.

[7] Koyama H, Noma S, Tamaki Y, Goto K, Kitamura E, Maeda T, et al. CT localisation of small pulmonary nodules prior to thorascopic resection: Evaluation of a point marker system. Eur J Radiol 2008;65:468-72. doi:10.1016/j.ejrad.2007.04.019.

[8] Mamatha J, Chaitra KR, Paul RK, George M, Anitha J, Khanna B. Cone Beam Computed Tomography-Dawn of A New Imaging Modality in Orthodontics. J Int Oral Health JIOH 2015;7:96-9.

[9] De Vos W, Casselman J, Swennen GRJ. Cone-beam computerized tomography (CBCT) imaging of the oral and maxillofacial region: A systematic review of the literature. Int J Oral Maxillofac Surg 2009;38:609-25. doi:10.1016/j.ijom.2009.02.028.

[10] Gupta J, Ali SP. Cone beam computed tomography in oral implants. Natl J Maxillofac Surg 2013;4:26. doi:10.4103/0975-5950.117811.

[11] Kang J, Huang J, Gailloud P, Rigamonti D, Lim M, Bernard V, et al. Planning evaluation of C-arm cone beam CT angiography for target delineation in stereotactic radiation surgery of brain 
arteriovenous malformations. Int $\mathrm{J}$ Radiat Oncol Biol Phys 2014;90:430-7. doi:10.1016/j.ijrobp.2014.05.035.

[12] Choi JW, Park CM, Goo JM, Park Y-K, Sung W, Lee H-J, et al. C-Arm Cone-Beam CT - Guided Percutaneous Transthoracic Needle Biopsy of Small (I $20 \mathrm{~mm}$ ) Lung Nodules: Diagnostic Accuracy and Complications in 161 Patients. Am J Roentgenol 2012;199:W322-30. doi:10.2214/AJR.11.7576.

[13] Hohenforst-Schmidt W, Zarogoulidis P, Vogl T, Turner JF, Browning R, Linsmeier B, et al. Cone Beam Computertomography (CBCT) in Interventional Chest Medicine — High Feasibility for Endobronchial Realtime Navigation. J Cancer 2014;5:231-41. doi:10.7150/jca.8834.

[14] Wang Z, Wu QJ, Marks LB, Larrier N, Yin F-F. Cone-beam CT localization of internal target volumes for stereotactic body radiotherapy of lung lesions. Int J Radiat Oncol Biol Phys 2007 ; 69:1618-24. doi:10.1016/j.ijrobp.2007.08.030.

[15] Pyone YY, Suriyapee S, Sanghangthum T, Oonsiri S, Tawonwong T. Determination of effective doses in image-guided radiation therapy system. J Phys Conf Ser 2016;694:012007. doi:10.1088/17426596/694/1/012007.

[16] Alvarado R, Booth JT, Bromley RM, Gustafsson HB. An investigation of image guidance dose for breast radiotherapy. J Appl Clin Med Phys 2013;14.

[17] Ohtaka K, Takahashi Y, Kaga K, Senmaru N, Kotani Y, Matsui Y. Video-assisted thoracoscopic surgery using mobile computed tomography: New method for locating of small lung nodules. J Cardiothorac Surg 2014;9:110. doi:10.1186/1749-8090-9-110.

[18] Gill RR, Zheng Y, Barlow JS, Jayender J, Girard EE, Hartigan PM, et al. Image-guided Video Assisted Thoracoscopic Surgery (iVATS) - Phase I-II Clinical Trial. J Surg Oncol 2015; 112:18-25. doi:10.1002/jso.23941.

[19] Narayanam S, Gerstle T, Amaral J, John P, Parra D, Temple M, et al. Lung tattooing combined with immediate video-assisted thoracoscopic resection (IVATR) as a single procedure in a hybrid room: our institutional experience in a pediatric population. Pediatr Radiol 2013; 43:1144-51. doi:10.1007/s00247-013-2665-6.

[20] Kim TH, Park CM, Lee SM, McAdams HP, Kim YT, Goo JM. Percutaneous transthoracic localization of pulmonary nodules under $\mathrm{C}$-arm cone-beam $\mathrm{CT}$ virtual navigation guidance. Diagn Interv Radiol Ank Turk 2016;22:224-30. doi:10.5152/dir.2015.15297.

[21] Schafer S, Otake Y, Uneri A, Mirota DJ, Nithiananthan S, Stayman JW, et al. Highperformance C-arm cone-beam CT guidance of thoracic surgery. In: Holmes III DR, Wong KH, editors., 2012, p. 83161I. doi:10.1117/12.911811.

[22] Uneri A, Nithiananthan S, Schafer S, Otake Y, Stayman JW, Kleinszig G, et al. Deformable registration of the inflated and deflated lung in cone-beam CT-guided thoracic surgery: Initial investigation of a combined model-and image-driven approach. Med Phys 2013;40:017501. 